Books, videos, cd-roms, dvds and any other relevant items submitted for a review in the BDJ should be addressed to:

Mike Grace, Editor, British Dental Journal, 64 Wimpole Street WIG 8YS

\section{Screening for oral cancer and pre-cancer in dental practice}

The Dental Channel, 2002

price $£ 59.99$

This new CD from The Dental Channel is one of a series; other subjects include dental anxiety, restoration of the root filled tooth, and endodontics. All these have a very similar format, and the familiarity this gives is most helpful.

On loading the $\mathrm{CD}$ you are presented with a screen giving you the serial number of your package, and inviting you to register your copy to unlock all the features. You will be able to run some parts of the package ten times prior to registration, following that you will be locked out for good.

The package is divided into four sections: references, case studies, information and links and finally the inevitable quiz. The quiz is a useful facility in allowing the user to assess their uptake of knowledge; this too forms the basis of the verified assessment. Negative marking is used, so simply clicking on random answers will not score too highly.

The case study section presents six cases. The first four are patients with suspicious lesions, and the remaining two demonstrate a full screening procedure and an excisional biopsy. Each of the first four cases invite you to interview the patient, make notes and come up with a diagnosis based on the answers given. You are then allowed to examine the patient with the aid of high quality clinical images, suggest investigations you may wish to carry out to confirm your findings and make your final diagnosis. There is also help on how an appropriate referral letter may be written.

The 'references' section gives more information relating to the conditions covered in this package, including screening aids, prevention and epidemiology. The 'information and links' section gives contact details and allows you to connect to the world wide web. The 'quiz' is self-explanatory.

I found this package easy to use, informative and enjoyable. This material, with its high quality images, video and sound, is a must for any dental practitioner who wishes to update his or her knowledge on oral cancer, in a relaxed, nonthreatening manner.

This package (along with the others in the series) is available to all GDPs working in the GDS free of charge, and may be obtained through the postgraduate deaneries. For those wishing to boost their verified CPD requirements there is an option to pay £59.99 (inc VAT) and obtain verified 'brownie points' from the comfort of the easy chair. For those purchasing the verified 'add on', I am assured that at $£ 52.00$ per hour, $£ 312.00$ worth of CPD may be delivered by this one $\mathrm{CD}$. Now that is a bargain.

D. Speechley

\section{Caring for your mouth}

The Dental Channel and The British Dental Health Foundation, 2002

price: $C D-R O M € 85.10+$ VAT;

$60 \mathrm{~min}$ video $£ 29.78+\mathrm{VAT}$.

Caring for your mouth is another excellent title produced by The Dental Channel, in association with the British Dental Health Foundation. Unlike the other CPD-based products that they have previously released, this is geared towards helping dentists, hygienists and oral health educators inform their patients about the essentials of oral healthcare in an uncomplicated yet effective manner. The title comes in both CD-ROM and educational video or DVD formats designed for use at the chair side or in the waiting room.

There is no doubt that patients respond well to explanations backed up by pictures. The use of the CD-ROM on a surgery computer to provide instant access to videos, pictures, text and narration takes patient education a step further. The messages put across are necessary for dental health education but can become monotonous if you are the dentist or hygienist repeating them many times a day. The CD-ROM allows the operator to quickly locate a relevant image or video using a search facility and display it to the patient with or without the accompanying narration as wished.

There is a 'Tell me about' section that can easily be browsed by the patient, giving answers to commonly asked dentally related questions. The $\mathrm{CD}-\mathrm{ROM}$ could also be run in a waiting room using an autopilot mode where the program plays in a loop over a 40-minute period.

The content of the program is detailed, concise and easy to digest. The pictures, videos and narration are unambiguous and every patient should find them easy to understand. All areas of oral health are covered in depth, including the messages required to educate all patients. From those patients with generally poor oral health needing a clear-cut explanation of why and how to clean their teeth and those needing a full explanation of the decay process and dietary factors, to the orthodontic patient who needs to be shown how to care for their new appliance.

The computer-generated animation showing the process of periodontal disease is particularly good, as is the section on cleaning aids. This section runs through all types of cleaning devices with accompanying videos, showing how to use them correctly. The only topic that warrants more attention is smoking and the damage it causes. The basic messages are there but pictures showing the hazards associated with smoking would make a more significant impact on the patient.

The ten short videos clips, on VHS and DVD, put across the important messages of oral healthcare, including why and how to brush, flossing, cleaning prostheses and looking after children's teeth. The total length of the video is 20 minutes and is ideal for the practice waiting room where it can be run on a loop. The videos are subtitled so the sound can be turned off when on continuous play. There is a 'home' version of the VHS tape, which can be sold or lent out to patients. This is especially useful for repetition of important messages. 

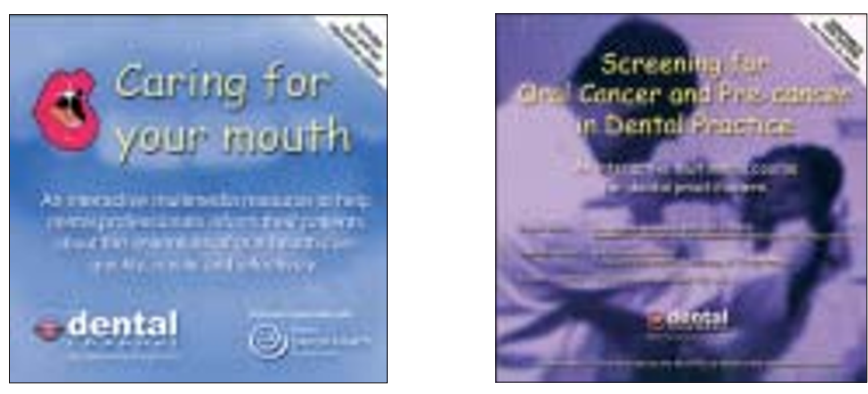
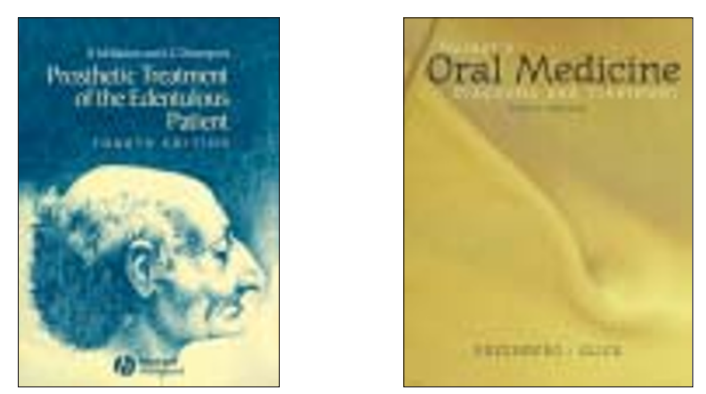

Caring For Your Mouth is an interesting and invaluable resource. The practicalities of dental health education are expressed far more effectively by using a visual, interactive medium such as this, rather than by verbal explanation alone. This package provides an essential aid to preventative care in the modern dental practice.

D. Jones

\section{Prosthetic treatment of the edentulous patient.}

R M Basker and J C Davenport

Oxford: Blackwell, 2002

price £29.25

ISBN 0632059982

In an age where there are, sadly, too few textbooks on complete denture prosthodontics it is nice to see a new edition of an old faithful. In this, the fourth edition of this book - the first was in 1976 - the authors seek to retain the same principles as in the three earlier editions, namely a biological approach (rather than a mechanistic one ) which is feasible, with an emphasis of good communication in 'team dentistry', all laudable tenets.

Chapter one deals with an overview of edentulousness, including demographics, limitations of complete dentures and general aspects of gerodontics. Chapter two concentrates on aspects influencing the outcome of treatment, principally those concerning the patient, the clinician and the technician. This chapter is disappointingly short and concentrates exclusively on patient-related aspects only.

Chapter three deals with the transition from the natural to an artificial dentition including overdentures. Here, as in other chapters, reference is made to evidence based research, which is qualitative and quantitative. As in previous textbooks, the value of this book would be increased by colour illustrations.

Chapter four deals with denture stability and there is some confusion in this chapter with retention, although the next chapter relates to jaw relations (theoretical aspects).

Chapters six to 14 deal with clinical aspects of complete dentures and these are of a good standard for undergraduate students, again colour photographs would have enhanced the text considerably.

Recall procedures and problem solving are dealt with in chapters 15 and 16, the latter is a welcome addition and I am sure will be appreciated by undergraduate readers. It includes aids to diagnosis of complete denture problems with some suggestions to detect them as well as how to avoid them.

In summation, the authors have achieved their aims and I would recommend this textbook for undergraduates. The lack of colour and the slightly dated form of the tables might not be attractive to graduates. F. McCord

\section{Burket's oral medicine: diagnosis and treatment.}

Greenberg and Glick 10th Ed.

New York: BC Decker Inc, 2003

price USD 99.95,

ISBN 1550091867

Lester Burket's textbook on oral medicine has been in print for five decades and this 10th edition celebrates the 50th anniversary of this classic text. Thirty authors are involved in bringing together an exhaustive compendium of information with major changes from previous editions. The book is divided into four parts, part one dealing with principals of diagnostics, parts two and three dealing with oral and orofacial conditions and part four focusing on oral aspects of systemic disease. Each disease has succinct descriptions. In part four medical diseases of interest are described in detail that concern medically compromised patients mostly seen in hospital practice. The book is accompanied by a CD-ROM.

The management of most orofacial lesions and conditions considered under various titles presents a well balanced overview, putting forward both sides of the arguments in those areas where there are many and deep controversies. Occasionally the authors' individual prejudices are allowed to shine through, but nevertheless the balance is generally very well done. In some sections however, treatment suggestions are limited, often simplified, without attention to evidence based therapies

There is some duplication of material; for example in chapter eight on oral cancer, Joel Epstein describes the management of oral leukoplakia when this has been covered in a preceding chapter on white and red lesions by Sol Silverman and co-authors. Salivary gland tumours are briefly mentioned again in chapter eight while the next chapter deals with this topic in more detail. Dr Epstein's chapter on oral cancer however, has a new dimension incorporating current molecular information useful to the readership.

Although there are plenty of illustrations, many continuing from early editions, the quality of illustration is unfortunately inferior to that of many other oral medicine texts. In the 21st century, a book of this calibre would have benefited from colour illustrations, particularly in view of the fact that it is the colour of the lesion that enhances many subtle morphological features often contributing to the correct diagnoses. This is particularly true for chapters on red, white and pigmented lesions. Despite being extremely well written, the reader will not find the illustrations useful to get a grasp of the clinical presentation.

A comprehensive bibliography at the end of each chapter ensures that full details of published cases, research or reviews are readily accessible.

There is some American bias in the way new information is set out, for example in the chapter on infectious diseases epidemiological data related to tuberculosis, hepatitis C infection, and AIDS all relate to United States only.

Family dental practitioners with enough shelf space for another reference text will find this book a useful purchase. It provides a very useful resource for oral medicine specialists and oral surgeons who have a particular interest in the pathology of the disorders they treat and the hospital trainees in many dental specialities preparing for their higher examinations. S. Warnakulasuriya 